\title{
Neoplasia quística del apéndice. Reporte de un caso clínico inusual en el Hospital Central Sur de Alta Especialidad PEMEX
}

Cystic neoplasm of the appendix. Report of an unusual clinical case at the South Central High Specialty Hospital PEMEX

Alfredo Chama-Naranjo*, Ulises Chávez-Villanueva y Jorge Farell-Rivas

Servicio de Cirugía General, Hospital Central Sur de Alta Especialidad, Ciudad de México, México

\section{Resumen}

Las neoplasias del apéndice son un grupo heterogéneo de tumores con una incidencia baja, la presentación clínica es inespecífica, cursando generalmente de forma asintomática, y la tomografía es el estudio de elección para el diagnóstico. El tratamiento dependerá del tipo histológico del tumor.

Palabras clave: Apéndice. Tumor mucinoso. Tumor mucinoso de bajo grado.

\begin{abstract}
The appendix neoplasms are a heterogeneous group of tumors with a low incidence, the clinical presentation is nonspecific, usually being asymptomatic, tomography is the study of choice for the diagnosis. Treatment will depend on the histological type of the tumor.
\end{abstract}

Key words: Appendix. Mucinous neoplasm. Low-grade appendiceal mucinous neoplasm.

\section{Introducción}

Las neoplasias del apéndice son un grupo heterogéneo de tumores, con una incidencia inferior al $0.5 \%$ de los tumores gastrointestinales ${ }^{1}$. La edad más frecuente de presentación es en la sexta década de la $v^{\text {vida }}{ }^{2}$. La clínica es inespecífica, predominando el dolor en la fosa iliaca derecha, por lo que a menudo se confunde con apendicitis aguda. El diagnóstico se realiza mediante histopatología ${ }^{3}$. Los tumores mucinosos representan el $8 \%$ de las neoplasias apendiculares. El riesgo es similar en ambos sexos, aunque algunos estudios demuestran un predominio en las mujeres ${ }^{4}$.

\section{Caso clínico}

Mujer de 43 años sin antecedentes de importancia para el padecimiento actual. Inicia 24 horas antes de su ingreso con dolor abdominal generalizado, acompañado de náuseas y vómitos. En la exploración física presenta dolor a la palpación en la fosa iliaca derecha, con McBurney positivo, sin datos de respuesta inflamatoria sistémica. La tomografía abdominal muestra un tumor dependiente del apéndice 
(Figs. 1 y 2). Se realiza laparotomía diagnóstica con resección de tumor apendicular (Fig. 3). Cuenta con reporte histopatológico de neoplasia quística mucinosa del apéndice de bajo grado, con bordes quirúrgicos negativos.

\section{Discusión}

La primera descripción de una neoplasia quística apendicular fue por Rokitansky en 1866, denominándola hydrops processus vermiformes ${ }^{5}$.

La clasificación de los tumores mucinosos del apéndice es controvertida, especialmente cuando carecen de malignidad pero presentan diseminación peritoneal de mucina. Los tumores confinados al apéndice son clínicamente benignos y se denominan de bajo grado; los que se diseminan por el peritoneo pueden tener diferente evolución clínica. Los tumores con invasión de la pared apendicular o alto grado de atipia evolucionan de forma más agresiva y se consideran adenocarcinomas ${ }^{6}$.

Según Pai y Longacre ${ }^{7}$, los tumores mucinosos del apéndice se clasifican en cistoadenoma mucinoso, neoplasia mucinosa de potencial incierto maligno, neoplasia mucinosa de bajo potencial maligno y adenocarcinoma mucinoso. La Organización Mundial de la Salud establece solo dos grupos, uno para los cistoadenomas y otro para las neoplasias apendiculares de bajo potencial maligno ${ }^{8}$. La tendencia actual es diferenciarlos por el grado histológico, según la clasificación del American Joint Committee on Cancer.

La forma de presentación es inespecífica, desde un cuadro asintomático la mayoría de las veces hasta cuadros similares a apendicitis aguda, tumor palpable o síntomas urológicos ${ }^{6}$. El diagnóstico clínico es difícil por el cuadro inespecífico; en general se encuentra como hallazgo incidental durante el procedimiento quirúrgico, un estudio de imagen o como resultado de la pieza quirúrgica ${ }^{9}$.

La tomografía se considera como la prueba de imagen de elección, la cual demuestra un tumor de baja densidad con pared delgada y en comunicación con el ciego ${ }^{10}$. En un estudio endoscópico se puede observar el signo del volcán, donde se aprecia un orificio con expulsión de moco. El tratamiento debe ser quirúrgico debido al potencial maligno y la prevención de las complicaciones: perforación del apéndice y diseminación ${ }^{11}$.

La cirugía puede variar desde la apendicetomía hasta la hemicolectomía derecha. Se recomienda

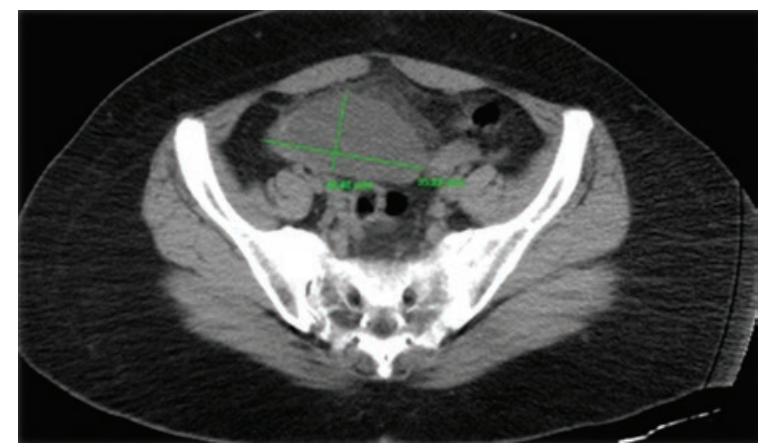

Figura 1. Tomografía en corte axial que muestra un tumor dependiente del apéndice.

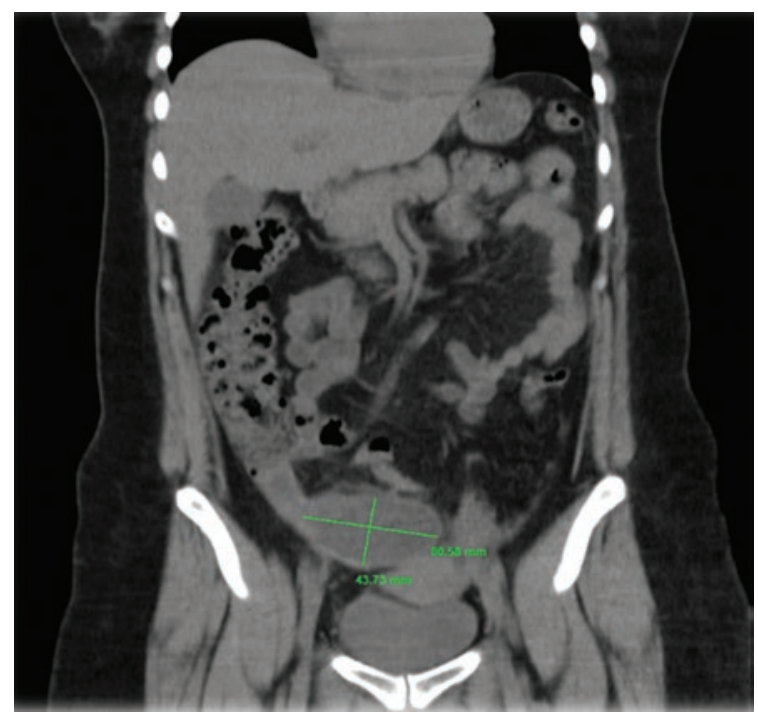

Figura 2. Tomografía en corte coronal en la que se determina el tamaño del tumor: $43 \times 80.3 \mathrm{~mm}$.

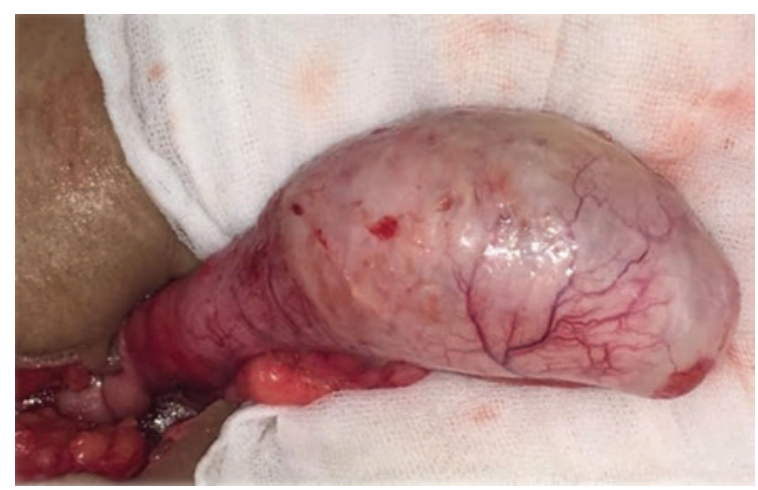

Figura 3. Tumor apendicular de aspecto quístico de $12 \times 8 \mathrm{~cm}$, forma ahusada, adherencias a pared de epiplón, líquido libre $(50 \mathrm{ml})$, de aspecto seroso.

realizar conversión a cirugía abierta si el abordaje comenzó laparoscópico debido al riesgo de diseminación de mucina y la necesidad de explorar áreas 
como el colon o los ovarios; sin embargo, algunos autores recomiendan terminar el procedimiento laparoscópico si se realiza una manipulación cuidadosa del tumor ${ }^{12}$.

Existe controversia sobre el manejo que debe hacerse. Si la lesión se encuentra confinada en el apéndice, la apendectomía será suficiente para resolver el problema. Si el apéndice está perforado, pero sin márgenes positivos ni invasión linfática, el tratamiento será apendicetomía más HIPEC (quimioterapia hipertérmica peritoneal). Si los márgenes son positivos, pero no hay afección ganglionar, debería realizarse una resección ileocecal más HIPEC. Si no se dispone de HIPEC, deberá llevarse a cabo un seguimiento estrecho de los pacientes ${ }^{13}$.

La hemicolectomía derecha se realiza para los adenocarcinomas mucinosos, debido al alto riesgo de metástasis linfáticas. Este procedimiento no debe realizarse sistemáticamente, sino que debe individualizarse para cada paciente ${ }^{14}$.

\section{Conclusión}

Los tumores mucinosos del apéndice pueden presentarse como un cuadro de apendicitis aguda o como resultado del análisis de la pieza quirúrgica. A pesar de que sigue sin haber una clasificación estandarizada y una nomenclatura, la Organización Mundial de la Salud propone clasificar a estos tumores en alto y bajo grado. La técnica quirúrgica dependerá de la clasificación histológica. Los pacientes con tumores de apéndice deberán tener un seguimiento estrecho con colonoscopias por el riesgo de asociación con tumores colorrectales.

\section{Agradecimientos}

Agradecemos a los médicos y residentes del hospital por hacer posible este artículo de reporte de caso.

\section{Conflicto de intereses}

Los autores no presentan conflicto de intereses.

\section{Financiamiento}

Los autores declaran que no se recibió financiamiento para realizar este artículo.

\section{Responsabilidades éticas}

Protección de personas y animales. Los autores declaran que para esta investigación no se han realizado experimentos en seres humanos ni en animales.

Confidencialidad de los datos. Los autores declaran que han seguido los protocolos de su centro de trabajo sobre la publicación de datos de pacientes.

Derecho a la privacidad y consentimiento informado. Los autores han obtenido el consentimiento informado de los pacientes y/o sujetos referidos en el artículo. Este documento obra en poder del autor de correspondencia.

\section{Bibliografía}

1. Benedix F, Reimer A, Gastinger I, Mroczkowski P, Lippert H, Kube R, et al. Primary appendiceal carcinoma. Epidemiology, surgery and survival: results of a German multicenter study. Eur J Surg Oncol. 2010;36:763-71.

2. Van den Heuvel MG, Lemmens VE, Verhoeven RH, de Hingh IH. The incidence of mucinous appendiceal malignancies: a population based study. Int J Colorectal Disease. 2013;28:1307-10.

3. Shaib W, Goodmanm M, Zhengjia C, Kim S, Brutcher E, Bekaii Saab T, et al. Incidence and survival of appendiceal mucinous neoplasm. A SEER analysis. Am J Clin Oncol. 2017:40:569-73.

4. Ruoff C, Hanna L, Zhi W, Shahzad G, Gotlieb V, Wasif Saif M. Cancers of the appendix: review of the literatures. ISRN Oncol. 2011;2011:728579.

5. Rouchad A, Gayet M, Bellin MF. Appendiceal mucinous cystadenoma. Diagn Interv Imaging. 2014;95:113-6.

6. Darriba-Fernández M, Madrozo González Z, Aranda-Dausa H, Sanjuan Darriga X, Hernandéz-Gañan J. Mucinous appendiceal neoplasm. Do we all speak the same language? Rev Esp Enferm Diag. 2012;104:44-5.

7. Pai R, Longacre T. Appendiceal mucinous tumors and pseudomyxoma peritonei: histologic features, diagnostic problems, and proposed classification. Adv Anat Phatol. 2005;12:291-311.

8. Misdraji J. Appendiceal mucinous neoplasms and pseudomyxoma peritonei. USA: Oncohema Key; 2016. Disponible en: https://oncohemakey. com/appendiceal-mucinous-neoplasms-and-pseudomyxoma-peritonei/

9. Lai CW, Yue CT, Chen JH. Mucinous cystadenocarcinoma of the appendix mimics acute appendicitis. Clin Gastroenterol Hepatol. 2013;11:e11.

10. Bravo Jaramillo EL, López Lucer WN, Chacha Vivar MJ, Machado Zamora JP, Romero Zhinin LA. Caso clínico: mucocele apendicular. Revista Médica HJCA [Internet]. 2017;9. (Consultado el 13 de febrero de 2019.) Disponible en: http://revistamedicahjca.iess.gob.ec/ojs/index.php/ HJCA/article/view/234/216.

11. Alemán Barrera RO, Catoira Martínez CA, Pando Pérez RM. Cistadenoma mucinoso de apéndice. Presentación de un caso. Medicent Electrón [Internet]. 2015;19(2). (Consultado el 22 de junio de 2018.) Disponible en: http://scielo.sld.cu/pdf/mdc/v19n2/mdc12215.pdf.

12. García A, Vázquez A, Castro C, Richart J, Gómez S, Martínez M. Mucocele of the appendix: presentation of 31 cases. Cir Esp. 2010;87:108-12.

13. Dixit A, Robertson J, Mudan S, Akle C. Appendiceal mucoceles and pseudomyxoma peritonei. World J Gastroenterol. 2007;13:2381-4.

14. Sugarbaker PH. Cytoreduction including total gastrectomy for pseudomyxoma peritonei - non operative management and biochemical findings. Br J Surg. 2002;89:208-12. 